\title{
Actualité et avenir de l'expertise scientifique dans le domaine des risques sanitaires de l'alimentation
}

\author{
Gérard PASCAL \\ Directeur de recherches honoraire à I'Inra
}

\begin{abstract}
The French and European structures of expertise in the field of the safety assessment of food evolved considerably during the last decade, like their work methods and the mode of selection of experts. The evolution at the international level is less perceptible. The conditions of exercise of expertise by the researchers, they also, changed: heavier load, multidisciplinarity, media and political pressures, criticisms of their impartiality. Only improvements of the conditions of exercise and of the judgment of these activities will lead to a stronger investment of the researchers of which it is however one of the missions.
\end{abstract}

Key words: expertise, research, food safety agencies, evaluation of scientists

merciaux ou de législations nouvelles (UE, $\mathrm{OCDE}, \mathrm{OMC} . .$.$) ; contractualisation et mise en$ œuvre des conventions internationales. Elle est ainsi devenue un exercice obligé, instrument dans la compétition internationale. Au-delà des nécessités économiques, l'expertise tend aujourd'hui à devenir l'épine dorsale de la décision publique, dans un contexte d'incertitudes sur certains risques sanitaires et environnementaux désormais appréhendés à l'échelle mondiale.

L'adoption et la mise en œuvre progressive (depuis le sommet de Rio en 1992) du principe de précaution repose sur la gravité, voire l'irréversibilité, des risques craints. Le principe de précaution nécessite le recours régulier à l'expertise car les mesures provisoires prises par I'autorité en cas de plausibilité forte du risque doivent être révisables, c'est-à-dire soumises à un examen périodique au regard des nouvelles données scientifiques acquises et de leur interprétation. En cas de plausibilité moyenne, le recours à la recherche s'impose d'emblée.

II ressort par ailleurs des débats récents que l'expertise scientifique ne doit pas se limiter à l'évaluation des risques sanitaires, mais qu'elle doit intégrer les aspects socio-économiques et l'évaluation du rapport risques/bénéfices. Les modalités d'intervention dans les processus d'évaluation des acteurs de terrain et plus largement de l'ensemble des acteurs d'une question, est l'objet de réflexions dans de nombreuses instances et dans des projets européens de recherche (par exemple Safefoods ${ }^{1}$ ).

La question des frontières entre l'expertise scientifique et la mise en œuvre des mesures de gestion, des compétences qu'elle mobilise, est également en discussion; la séparation formelle entre évaluation et gestion de risques

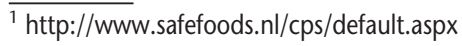

impose en même temps des échanges très construits entre évaluateurs et gestionnaires. L'expertise scientifique, quand elle concerne des sujets vastes, ne peut plus se concevoir comme individuelle mais elle doit désormais être pratiquée collectivement, dans le souci d'abord d'associer les disciplines scientifiques concernées, mais également avec la préoccupation de diversifier les approches et points de vue favorisant la contradiction, la mise en lumière des controverses, c'est-à-dire de rassembler tous les éléments pertinents pour éclairer la décision. En ce sens, l'expertise judiciaire faisant appel à un expert unique est largement fragilisée.

Mon propos ne traitera donc que de l'expertise pratiquée uniquement dans des collectifs organisés, par des individus appartenant essentiellement au secteur public.

\section{Évolution des structures pratiquant l'expertise dans le domaine sanitaire}

\section{Au plan national}

J'ai commencé modestement à m'impliquer dans des activités d'expertise au sein d'une commission du Centre national d'études et de recherches sur la nutrition et l'alimentation du CNRS (Cnerna), dans les années 70. Nous avions, au sein d'un groupe d'une quinzaine de chercheurs, préparé un projet de méthodologie d'évaluation de la sécurité des additifs alimentaires, destiné à la section de l'alimentation du Conseil supérieur d'hygiène publique de France (CSHPF). Ce travail, réalisé sur plusieurs années, en toute sérénité, à l'abri de toute pression médiatique, a permis de souder un groupe de chercheurs qui ont gardé des contacts de très nombreuses années. II a été publié sous la forme d'un numéro spécial d'une revue scientifique, les Annales de la nutrition et 
de l'alimentation. II a ensuite été discuté au CSHPF, a fait l'objet d'un avis très argumenté qui a lui-même conduit à une circulaire du 8 août 1980 sur la présentation des dossiers de demande d'autorisation d'additifs alimentaires.

C'est ainsi que s'élaborait la réglementation et que se préparaient, se formaient, les futurs experts du CSHPF dont certains allaient ensuite "monter» au niveau international dans le domaine de l'alimentation humaine. Une filière analogue existait pour l'alimentation animale, avec une Commission interministérielle et interprofessionnelle de l'alimentation animale (CIIAA). En fait, le système était plus complexe car il existait d'autres comités et commissions dans les domaines des produits diététiques, des produits phytosanitaires, des technologies alimentaires, etc.

II était devenu tellement obscur dans sa complexité que les pouvoirs publics ont décidé de le simplifier et de le rationaliser, en créant, à I'occasion de l'adoption de la loi de sécurité sanitaire du $1^{\text {er }}$ juillet 1998, une Agence nationale de sécurité sanitaire des aliments (Afssa) regroupant l'ensemble des structures d'expertise dans le domaine de l'alimentation. Enfin, pas tout à fait toutes les structures puisque les produits phytosanitaires y avaient échappé, anomalie aujourd'hui corrigée.

\section{Au plan européen}

Un comité scientifique de I'alimentation humaine (SCF) a été mis en place au sein de la Commission européenne en 1974, pour répondre aux questions qui concernaient la sécurité sanitaire des aliments de l'homme. Un comité scientifique de l'alimentation animale (Scan) a également été créé, de même qu'un comité scientifique vétérinaire (SVC). Ces comités étaient placés au sein de directions générales (DG) de la Commission européenne en charge de la gestion des risques correspondants, DC industrie et marché intérieur pour le premier, DG agriculture pour les suivants. Cette structure est restée en place jusqu'à ce que la crise dite de « la vache folle » éclate en 1996. Cette crise au sein de la Commission a conduit à la réorganisation des comités scientifiques [2] et en particulier à leur regroupement au sein d'une DG XXIV, «Politique des consommateurs et protection de leur santé » (devenue aujourd'hui DG Sanco, pour Santé et Consommation) qui permettait une séparation claire de l'évaluation des risques et de leur gestion. Les nouveaux comités scientifiques $[3,4]$ et en particulier le comité scientifique directeur (SSC) ont émis de nombreuses opinions de 1997 à 2003, disponibles sur le site Internet de la DG Sanco. C'est ainsi que le SSC a émis 260 opinions sur les questions de la vache folle et des prions à la demande de la Commission, opinions qui ont participé à la maîtrise de la maladie animale et à la réduction du risque d'exposition de l'homme au prion bovin.

Cette situation annonçait l'organisation définitive de l'expertise européenne dans le domaine de la sécurité sanitaire des aliments, arrêtée dans le règlement $E C N^{\circ} 178 / 2002$ [5] du Parlement et du Conseil. Ce règlement instituait l'Autorité européenne de sécurité des aliments (Efsa), autorité indépendante créée au centre du triangle Commission, Parlement, Conseil, comme recommandé dans un rapport préparatoire [6] rédigé à la demande du directeur général de la DG XXIV de l'époque, Horst Reichenbach. La séparation entre l'évaluation et la gestion des risques était ainsi définitivement réalisée. II reste à assurer la bonne coordination entre ces quatre partenaires de l'analyse de risque.

Ainsi, I'organisation de l'expertise au niveau I'Union européenne a connu de profonds bouleversements au cours des 10 dernières années.

\section{Au plan international}

Il n'en est pas de même au plan international. Les comités scientifiques de la Commission du Codex alimentarius, Comité mixte FAO/OMS d'experts des additifs alimentaires (Jecfa) et Réunions conjointes FAO/OMS sur les résidus de pesticides (JMPR), n'ont pas connu de réforme majeure, ni dans leur fonctionnement, ni dans les moyens dont ils sont dotés au cours des dernières décennies.

\section{Les modalités de fonctionnement des instances d'expertise}

\section{Le choix des experts}

Le premier point qui mérite attention est bien celui du choix des experts dont les règles n'ont pas toujours été très claires.

Pour ce qui concernait la section de l'alimentation du CSHPF, c'est la Direction générale de la santé qui décidait de la composition de la section, en tenant compte des propositions qui pouvaient être faites par ses membres et de ses critères propres connus d'elle seule. Les membres étaient ensuite nommés par arrêté ministériel. Depuis la création de l'Afssa, les membres des comités scientifiques spécialisés sont nommés par arrêté interministériel, sur proposition du directeur général, après sélection des candidats sur dossier adressé à l'agence en réponse à un appel à candidature largement diffusé. Les critères de choix sont explicités dans l'appel à candidature et des consignes précises données aux jurés responsables de la notation des dossiers. Le conseil scientifique de I'Afssa est ensuite consulté et donne son avis au directeur général. À l'occasion du processus de sélection, des listes d'experts non retenus pour faire partie des comités, mais dont les compétences sont reconnues, sont établies pour alimenter des groupes de travail.

Les membres des comités scientifiques européens, avant 1997, étaient désignés par les DG de la Commission après consultation des États membres qui étaient invités à proposer des listes « longues » de candidats potentiels. Dans les faits, certains États proposaient des listes " courtes», c'est-à-dire comportant strictement le nombre de membres qui étaient attribués à chacun. C'était ainsi les États qui choisissaient en fait les membres des comités. La crise de la "vache folle» de 1996, qui a entraîné la réorganisation des comités scientifiques, a aussi permis de modifier les modalités de désignation de leurs membres. C'est bien I'Europe qui a montré le chemin à la France puisque les modalités adoptées en 1999 par I'Afssa ont été copiées sur celles mises en place en 1997 par la Commission. Appel à candidature, notation des dossiers par des jurys présidés par des scientifiques de renom (parfois un prix Nobel), établissement d'une «liste longue » de candidats reconnus aptes et choix final par la Commission sur la base d'équilibres délicats en matière de genre, de spécialisation scientifique, d'origine géographique, voilà quel a été le processus de nomination de 1997 à 2003. Depuis, la responsabilité de la sélection revient à l'Efsa, qui a retenu une démarche analogue à celle de la Commission. Comme à l'Afssa, des listes d'experts compétents dans différents domaines, mais pas retenus pour siéger comme membres des panels, sont établies grâce au processus d'appel à candidature. Ils pourront être appelés en fonction des besoins pour siéger dans des groupes de travail des panels.

En France et à Bruxelles, les modalités de désignation des experts des comités scientifiques se sont considérablement améliorées et un peu plus de transparence introduite. Le mouvement a été beaucoup plus lent au niveau international, c'est-à-dire dans les comités scientifiques du Codex. Ce n'est que depuis peu d'années que des appels à candidature sont publiés pour constituer des listes (rosters) d'experts susceptibles de siéger dans ces comités ou de participer à des consultations FAO/OMS sur des sujets spécifiques. Ceci est une amélioration incontestable, même si les règles de choix au sein de ces listes restent parfois obscures. Mais il a y un aspect nettement plus critiquable dans la constitution du groupe de participants aux réunions. Nous invitons le lecteur à consulter la liste des participants aux réunions du Jecfa; il constatera que, outre les membres des groupes FAO et OMS du comité, figure une représentation très, 
voire plus importante des secrétariats. Un examen encore plus attentif de la représentation du secrétariat de l'OMS pourra laisser planer un doute sur l'indépendance de certains de ses membres et conduire à s'interroger sur les pressions dont le comité pourrait être potentiellement l'objet.

\section{La question des conflits d'intérêt}

Cette question n'a été sérieusement abordée que depuis environ 10 ans, au niveau européen, sur le modèle britannique. Après la mise en place des nouveaux comités de la Commission en 1997, des déclarations générales annuelles d'intérêt ont été progressivement imposées aux membres et des déclarations plus spécifiques en fonction de l'ordre du jour, exigées par le président à l'ouverture de chaque séance à partir de 2000. Ce processus perdure évidemment dans les panels de l'Efsa.

Dans ce domaine également, l'Afssa s'est alignée sur le modèle européen.

Les déclarations annuelles en France et à Bruxelles sont maintenant publiques et consultables sur les sites Internet des agences.

\section{Les moyens mis à disposition des comités d'experts}

Ces moyens étaient notoirement insuffisants au CSHPF et les agents en charge du secrétariat ont travaillé toujours en limite de rupture pour assurer des réunions mensuelles. Les experts ne pouvaient attendre aucun soutien matériel (recherche de documents, bibliographie, etc.) pour les aider dans leur tâche. La création de I'Afssa a donné aux comités d'experts spécialisés des moyens matériels et humains considérablement améliorés.

C'est avec soulagement que j'ai pu à partir de 1986, travailler dans de bien meilleures conditions qu'en France, dans les comités européens qui ont disposé progressivement de moyens plus importants. J'y ai rencontré des secrétaires scientifiques de grande qualité, ayant souvent à la fois une expérience de chercheur et de gestionnaire scientifique. Quelques banques de données ont facilité notre tâche, qui était extrêmement lourde au SSC qui se réunissait au minimum 2 jours par mois.

Le secrétariat du Jecfa a aussi été assuré par des équipes expérimentées. Leur tâche était cependant plus facile, le JECFA ne se réunissant le plus généralement que 2 fois par an sur des périodes de plusieurs jours.

\section{Les rôles respectifs de l'expertise interne et des experts des comités}

On touche là un aspect délicat du fonctionnement des instances d'expertise. Un volet important concerne la façon dont les responsables de ces instances considèrent les experts. II m'est arrivé d'entendre, c'était certes il y a longtemps, une responsable de la DGS assurer que les experts sont tellement fiers de siéger au CSHPF qu'il ne faudrait pas qu'en supplément ils réclament de la considération. Opinion personnelle sans doute, mais j'ai cependant l'impression d'avoir ressenti un sentiment assez proche dans d'autres instances dont les agents oublient sans doute que sans experts ils devraient se trouver un autre emploi ! À moins qu'ils n'estiment qu'ils puissent très bien assurer la mission d'expertise en appui à la décision publique en interne, sans recourir à des experts externes «incontrôlables". Voilà un piège dans lequel il ne faut surtout pas tomber : les secrétariats de comité doivent savoir quelles sont les limites de leur mission et quelles sont les responsabilités des experts. La responsabilité des opinions scientifiques émises revient à ces derniers et c'est heureux. Je reviendrai sur cet aspect plus loin.

\section{La transparence et la communication}

Elles constituent aussi des aspects fondamentaux des activités des instances d'expertise. Les membres du CSHPF se sont battus pendant des décennies pour obtenir la publication des avis, qui était décidée au coup par coup, dans de rares circonstances, par le cabinet du ministre de la Santé. Le législateur a lui, récemment, imposé la publication des avis de l'Afssa. II aurait bien fait d'aller plus loin et d'imposer la publication de tous les avis scientifiques des comités d'experts spécialisés.

C'est dès 1997, que les avis des comités européens et les comptes rendus de leurs réunions ont été publiés sur Internet dès leur adoption. Les rapports du Jecfa sont également publiés, mais avec un certain retard en raison d'une publication papier, inconvénient pallié en partie seulement grâce à l'informatique. On peut ainsi dire qu'aujourd'hui la transparence est assez correctement assurée, à un niveau qui respecte un minimum de confidentialité des débats dans les stades qui précèdent l'adoption des avis.

Les rapports qui servent de base aux opinions émises ne sont souvent pas publiés dans la littérature scientifique et les noms de leurs auteurs ne sont pas suffisamment mis en évidence. Ceci nuit à la reconnaissance de leurs activités.

\section{Et les experts dans tout ça ?}

Il fallait, je crois, planter le décor avant d'en arriver à évoquer la situation des experts et les obstacles à l'exercice de leurs activités d'expertise.

\section{La situation des experts}

Les trois grands principes destinés à assurer la légitimité des experts ont été rappelés lors de la réorganisation des comités européens : excellence, transparence et indépendance.

L'excellence académique devrait être assurée par les procédures de sélection actuelles mais elle ne peut constituer le seul critère. Une expérience de terrain dans certains domaines, qui ne fait pas l'objet de publications systématiques, pourra être appréciée. L'aptitude à travailler en groupe, à accepter le débat contradictoire puis à contribuer à l'émission d'avis utiles au gestionnaire de risques sans rester en permanence sur la réserve qui est la posture du chercheur dans son laboratoire, sont des critères à prendre en compte. Ces qualités doivent aussi être reconnues par l'établissement dans lequel l'expert exerce ses activités de recherche. C'est une question qui est au cœur de la situation de l'expert. Si des critères, certes critiquables, existent pour juger de l'excellence de l'activité académique des chercheurs comme la liste et la qualité de leurs publications, il est plus difficile de proposer des critères pour juger de la qualité de leurs activités d'expertise. L'évaluation individuelle des chercheurs, par des commissions scientifiques spécialisées ou au moment des concours organisés pour leur promotion, est réalisée par des jurys le plus souvent constitués d'universitaires pour lesquels les critères académiques sont pour beaucoup les seuls à retenir. Même dans les organismes de recherche finalisée, une espèce de sentiment d'infériorité conduit à se ranger aux systèmes d'évaluation dits de l'excellence ! Si une telle situation perdure, aucun chercheur ne sera incité à participer, pour une part de son activité, à un travail d'expertise. Elle donnera raison à ceux qui pensent que seuls ceux qui ne sont pas en mesure d'être reconnus sur la base des critères traditionnels ont assez de temps libre pour se consacrer à l'expertise.

La transparence, qui concerne la traçabilité des débats entre experts, la publication des résultats de l'expertise, la mention des avis minoritaires et des controverses, a été déjà évoquée ci-dessus.

L'indépendance, ne devrait-on pas dire I'impartialité des experts, renvoie au principe de la séparation entre l'évaluation et la gestion et exige des experts qu'ils ne soient pas liés par des intérêts politico-économiques, professionnels ou personnels, qui pourraient influer sur leur travail d'évaluation, ou, à tout le moins, que ces intérêts soient déclarés comme nous l'avons déjà évoqué, à charge pour le commanditaire d'apprécier si ces intérêts éventuels sont compatibles avec la liberté nécessaire de jugement de l'expert. II est clair que cette question est au cœur de vives controverses; les exemples sont nombreux, les plus récents concernent les OGM et les produits phytosanitaires. La question des conflits d'intérêt vient empoisonner les activités des experts émanant du 
secteur public. Beaucoup de ceux qui participent à des comités organisés par les pouvoirs publics sont accusés d'être à la solde du pouvoir économique, souvent parce qu'ils ont des relations avec les professionnels. Mais comment peut-on pratiquer une expertise sans connaître la réalité du terrain qui permet d'identifier plus précisément les dangers? Voudrait-on que n'existe qu'une expertise « en chambre »? Quelle est cette société que certains nous proposent, dans laquelle on devrait considérer une partie des acteurs comme des lépreux dont il ne faut pas s'approcher? La transparence des relations entre experts et acteurs de la chaîne alimentaire est l'élément essentiel; les accusateurs ne remplissent d'ailleurs pas, à ma connaissance, de déclaration d'intérêt publique. C'est très dommage car nous pourrions alors juger de leur degré $d$ 'indépendance, les intérêts pouvant être du domaine économique, mais aussi politique, administratif, intellectuel (activités public interest groups, professional/scientific societies, religious organisations, communication media, etc.), comme le considère l'Efsa pour la rédaction des déclarations d'intérêt de ses experts. Les attaques de plus en plus violentes portées contre l'intégrité des experts, qui émanent de certains médias et de groupes d'activistes, découragent les plus jeunes qui ont tendance à se réfugier dans le calme et la quiétude de leur laboratoire, mais n'assument alors plus l'une des misions du chercheur.

Outre la considération nécessaire des autorités commanditaires pour leurs experts, l'appui moral et juridique si nécessaire, matériel et humain à leurs activités est un élément majeur de progrès. La charge de travail des comités scientifiques s'est considérablement accrue, les questions posées se sont complexifiées (exemple des prions et de la vache folle, des nanotechnologies, etc.), le climat dans lequel les opinions sont attendues s'est dégradé, la pression des «politiques» s'est accentuée. Tout ceci rend encore plus nécessaire l'appui documentaire, d'information, de secrétariat, qui permettra aux chercheurs d'économiser un maximum de temps.

Si les conditions du travail d'expertise doivent impérativement être rationalisées et un minimum d'assurance qualité garantie, la contrainte ne doit pas être telle qu'elle éloigne les meilleurs chercheurs des activités d'expertise. J'ai été profondément choqué que des individus n'ayant aucune expérience de la pratique de l'expertise, en particulier internationale, se mêlent d'imposer une normalisation de l'expertise qui repose sur des pages de contraintes. Combien d'experts de qualité ontils été découragés par le risque d'application aveugle de consignes incompatibles avec des activités exercées souvent dans des situations $d^{\prime}$ incertitude scientifique qui imposent un jugement personnel de l'expert qui ne se « normalise » pas?

\section{Comment reconstruire et développer une force d'expertise en France?}

La situation est grave, mais pas désespérée! Une relève des plus anciens existe, aux plans national, européen et international. Mais le temps passe et les moins anciens vieillissent. Le nombre de candidatures déposées en réponse aux appels ne croît pas, bien qu'il ait été satisfaisant lors du dernier appel à candidature de l'Efsa, alors que la demande augmente considérablement. Or, en France, l'expertise en appui à la décision publique fait partie des missions des chercheurs et enseignantschercheurs et des organismes de recherche qui auront à rendre compte de leur activité dans ce domaine. Le besoin d'expertise est souligné au plan international, les conflits se réglant en partie sur la base d'avis scientifiques. Il devient impératif de mettre en place les conditions $d^{\prime}$ 'une pratique acceptable de l'expertise par les meilleurs scientifiques. Cette exigence n'est pas nouvelle ; le Professeur Pierre Louisot, alors président de la section d'alimentation du CSHPF, président du Cnerna et de I'IFN, n'a-t-il pas, il y a plus de 10 ans, saisi les ministres concernés de l'urgence de la question? Le sénateur Claude Saunier n'a-t-il pas rappelé en 2005 l'importance du problème dans son rapport sur la mise en œuvre de la loi du $1^{\text {er }}$ juillet 1998 à l'Office parlementaire d'évaluation des choix scientifiques et technologiques [7] ? N'at-il pas organisé, avec le député Jean-Yves Le Déaut, le 6 décembre 2005, une réunion à I'Assemblée nationale consacrée à l'expertise ${ }^{2}$ et présenté une proposition de loi sur « l'organisation et la valorisation de l'expertise publique »? Depuis rien n'a bougé ou pratiquement rien! Fait-il en conclure que "l'enjeu n'en valait pas la chandelle »? La présence d'experts britanniques, néerlandais, américains n'a fait que croître dans les comités internationaux (il n'y a que 2 Français dans le « roster » des experts en sciences biologiques du Jecfa). Si l'on veut renverser la tendance, les conditions suivantes devraient être remplies :

- Organiser dans les universités et les organismes une sensibilisation aux activités d'expertises qui font partie des missions du chercheur et de l'enseignant-chercheur et de leurs organismes [8] et des formations spécifiques, par exemple sous la forme d'écoles de chercheurs. II s'agit aussi de leur faire prendre conscience

\footnotetext{
${ }^{2}$ (http://www.assemblee-nationale.fr/12/rapoff/i2890.asp)
}

de leur rôle et des limites de ce rôle d'évaluateur, mais dans le même temps de les mettre en garde vis-à-vis des tentatives d'utilisation, voire de manipulation dont ils pourraient faire l'objet de la part des pouvoirs divers (économique, politique, administratif, associatif, etc.) au milieu desquels ils seront amenés à évoluer.

- Introduire dans leurs organismes d'appartenance, une procédure de gestion des conflits d'intérêt qui assure une crédibilité en matière d'impartialité et limite les critiques, le plus souvent injustifiées, dont leur indépendance fait de plus en plus souvent l'objet.

- Concrétiser dans les organismes et les universités le lien expertise/recherche pour ce qui a trait à l'évaluation et au retour de l'activité $d^{\prime}$ expertise sur la programmation de la recherche. Cette activité devrait être évaluée par des jurys comprenant des membres capables de juger de l'importance de ces activités pour la décision publique et s'appuyant sur des critères de jugement qui restent à définir par les structures d'évaluation. Le retour de l'expertise vers la recherche doit être organisé ; il montrera comment l'expertise permet d'identifier des lacunes des connaissances qui sont susceptibles de permettre de nouvelles voies de recherche et de proposer des projets d'envergure internationale (projets européens dans le domaine des prions et des OGM par exemple). À l'inverse, les activités de recherche viennent en permanence enrichir les connaissances et l'expérience des experts. Cet aller-retour permanent vient justifier l'avis selon lequel l'expert doit « baigner » dans la recherche.

- Faciliter le travail d'expertise en dégageant les moyens humains et matériels nécessaires et en établissant un climat de respect mutuel favorisant une étroite collaboration entre les experts scientifiques et les agents des structures qui organisent l'évaluation. L'expertise interne disponible dans ces structures doit se concentrer sur la mise en place d'outils communs comme des banques de données (composition des aliments, consommation alimentaire, etc.) ou des approches transversales comme l'appui statistique, qui viennent en soutien du travail des experts. Elle ne doit surtout pas se substituer à l'expertise externe et prendre ainsi le risque de pratiquer une expertise "en chambre » déjà évoquée. Les contraintes liées à la rigueur, à la transparence et à l'assurance qualité de l'expertise doivent être réduites au maximum pour ne pas décourager les chercheurs dont l'expertise ne représente qu'une partie de leur activité, qui sont déjà trop contraints à des tâches bureaucratiques qui n'ont rien à voir avec leur métier et dont le temps est compté. - Les experts scientifiques devraient être étroitement associés aux discussions entre les agences et les décideurs administratifs et politiques afin de bien comprendre les attentes de ces 
derniers et de leur exposer les arguments qui les ont conduits à adopter leurs avis. Ils doivent aussi pouvoir participer à des discussions plus larges, dans un «second cercle " comme le Conseil national de l'alimentation (CNA) qui réunit tous les acteurs de la chaîne alimentaire et dont les avis doivent venir éclairer la décision publique en mettant en perspective les avis scientifiques par rapport aux autres aspects d'une question.

- Toutes les améliorations qui précèdent contribueraient à accroître l'intérêt pour les chercheurs des activités d'expertise et à attirer les meilleurs vers ces activités. Elles clarifieraient également les rôles et responsabilités de chacun, sans mélange de genre. L'expert a la responsabilité de l'évaluation, mais sûrement pas la légitimité pour se mêler en quoi que ce soit de gestion. Le décideur n'a pas à suivre en aveugle les avis scientifiques, mais devrait expliquer de manière transparente, s'il s'en écarte, les raisons de ses choix de gestion et les aspects politiques, sociaux, économiques, commerciaux, éthiques, etc. auxquels il a donné la priorité.

\section{RÉFÉRENCES}

1. ROQUEPLO P. Entre savoir et décision, l'expertise scientifique. Science en question. Paris: INRA, 1997.

2. EUROPEAN COMMISSION. Commission Communication on Consumer health and food safety, COM (97) 183 fin. of 30 April 1997.

3. EUROPEAN COMMISSION. Commission Decision No97/404/EC of 10 June 1997 (Official Journal N'L 169 of 27.06.97).

4. EUROPEAN COMMISSION. Commission Decision N²97/579/EC of 23 July 1997 (Official Journal $N^{\circ} L 237$ of 28.8.97).

5. EUROPEAN PARLIAMENT AND COUNCIL. Regulation (EC) N ${ }^{\circ} 178 / 2002$ of the European Parliament and of the Council of 28 January
2002 laying down the general principles and requirements of food law, establishing the European Food Safety Authority and laying down procedures in matter of food safety.

6. JAMES WPT, KEMPER F, PASCAL G, (December 1999), A European Food and Public Health Authority - The future of scientific advice in the EU, (htpp://ec.europa.eu/food/fs/sc/future en.html).

7. SAUNIER C. L'application de la loi n ${ }^{\circ} 98-535$ du 1er juillet 1998 relative au renforcement de la veille sanitaire et du contrôle de la sécurité sanitaire des produits destinés à l'homme, Rapport à l'Office parlementaire d'évaluation des choix scientifiques et technologiques, (http ://www.assemblee-nationale.fr/12/rapoff/i2890.asp, 15 février 2005).

8. Loi de programme $n^{\circ} 2006-450$ du 28 avril 2006 pour la recherche, articles 14 et 15 , J.O. du 19 avril 2006, page 5820 . 